\title{
XPS measurements for probing dynamics of charging
}

\author{
Sefik Suzer ${ }^{\mathrm{a}, \mathrm{b}, *}$, Hikmet Sezen $^{\mathrm{a}, \mathrm{b}}$, Gulay Ertas ${ }^{\mathrm{a}, \mathrm{b}}$, Aykutlu Dâna ${ }^{\mathrm{b}}$ \\ a Department of Chemistry, Bilkent University, 06800 Ankara, Turkey \\ ${ }^{\mathrm{b}}$ Institute of Materials and Nanotechnology, 06800 Ankara, Turkey
}

\section{A R T I C L E I N F O}

\section{Article history:}

Available online 13 February 2009

\section{Keywords:}

Differential charging

Dynamical XPS

Dielectric properties

Resistance

Capacitance measurements using XPS

\begin{abstract}
A B S T R A C T
The technique of recording X-ray photoemission data while the sample rod is subjected to $\pm 10.0 \mathrm{~V}$ (dc) or square-wave pulses (ac) with varying frequencies in the range of $10^{-3}$ to $10^{3} \mathrm{~Hz}$ for probing charging/discharging dynamics of dielectric materials, is reviewed. Application of this technique introduces charging shifts as well as broadening of the peaks, which depend non-linearly on the polarity, as well as on the frequency of the pulses applied. These changes have been measured on: (i) an artificially created dielectric sample consisting of a Au metal strip connected externally to a series resistor of $1 \mathrm{M} \Omega$ and a parallel capacitor of $56 \mathrm{nF}$, and two real dielectric films; (ii) a $20 \mathrm{~nm}$ organic polystyrene film spin-coated on a silicon substrate; (iii) a $10 \mathrm{~nm} \mathrm{SiO}{ }_{2}$ inorganic layer thermally grown on silicon. A simple circuit model is introduced to simulate the charging shifts and the peak broadenings. Although this simple model faithfully reproduces the charging shifts in all three cases, and also some of the broadenings for the artificial dielectric and the polystyrene film, the additional broadening in the negatively charged peaks of the $\mathrm{SiO}_{2}$ dielectric film cannot be accounted for. It is also claimed that these experimental findings can be used for extracting material-specific dielectric properties.
\end{abstract}

(c) 2009 Elsevier B.V. All rights reserved.

\section{Introduction}

Control of charge accumulation and dissipation (charging/discharging) in dielectric materials is the essence of the rational design of various devices and sensors [1]. This is especially important for $\mathrm{SiO}_{2}$ as the thickness of the dielectric layer is expected to shrink down to few atomic layers for the next generation of metal-oxide-semiconductor (MOS) devices [2]. On the other hand, organic dielectric materials, are also important class of materials utilized for various applications, and here again, charge accumulation/dissipation is pivotal for their advanced functionalities [3-5]. Accumulation of charge in a dielectric layer occurs via various trapping mechanism(s) and is normally probed by electrical current-voltage and/or current-capacitance measurements [6-9]. Photoemission, utilizing UV, X-rays and lasers, has also been employed for probing very fast $\left(<10^{-9} \mathrm{~s}\right)$ charging dynamics [10-12]. Core-level X-ray photoemission, XPS, is especially attractive since additional chemical information can also be derived from the line positions of the corresponding peaks. However, the measured line positions are altered by local potentials developed, due to the uncompensated charges resulting from photoelectron emission, especially for poorly conducting samples or regions (layers or

\footnotetext{
* Corresponding author at: Department of Chemistry, Bilkent University, 06800 Ankara, Turkey. Tel.: +90 312 2901476; fax: +90 3122664068.

E-mail address: suzer@fen.bilkent.edu.tr (S. Suzer).
}

domains) within such samples [13-19]. Excellent charge compensation methods have been developed using low-energy electrons, ions and/or photons [20,21].

One can also utilize XPS for understanding the mechanisms leading to and/or controlling of the charging/discharging processes in materials, which offer great possibilities for researchers in all fields [22]. Several applications have been reported, utilizing the charging, called controlled surface charging, for extracting chemical, physical, structural, and electrical parameters of various surface species [23-28]. For example, it was shown that monolayer films are not affected, but multilayer films are affected by the voltage applied $[29,30]$. Recently, using silica-coated gold substrates secondary electron emission control in XPS has been shown by employing sample biasing and a top retarding grid to control the photoelectron current [31]. Islam and Mukherjee have used the positive charging to derive information about the structure of Langmuir-Blodgett films of cadmium arachidate on silicon substrate [32]. Using a slightly different strategy, and by applying voltage stress to the sample while recording XPS spectra, we have shown that the extent of charging can be controlled via which a range of analytical and electrical information could be extracted [33-36]. In addition to static information derived from application of dc voltage stress, dynamical information can also be extracted if the voltage stimuli is applied in the form of pulses, as we have recently reported [37-42].

In this contribution, we review our recent investigations for analysis of XPS data, recorded by applying the voltage stress in the form of bipolar square-wave (SQW) pulses for probing 
charging/discharging dynamics of thin dielectric surface structures, and we also supplement our experimental data with a theoretical model. Previously reported dynamical photoemission measurements have been either in the ultra-fast, sub-pico-seconds regime using laser excitations [10-12], or in a much longer time regimes ( $10^{1}$ to $10^{4} \mathrm{~min}$ ) with conventional XPS measurements [17,24,43-45]. Our measurements fill this gap and provide information on charging/discharging dynamics of dielectric materials in the range of $10^{-3}$ to $10^{3} \mathrm{~s}$, matching those of many chemical-biochemical processes [46].

\section{Experimental}

For the case of inorganic dielectric material we have chosen a ca. $10 \mathrm{~nm}$ silicon oxide layer grown thermally on HF-cleaned Si (1 00 ) substrates at $500^{\circ} \mathrm{C}$ in air. For organic dielectric we have used a thin film (20-60 nm thick) of polystyrene (PS) spin-coated on a clean silicon substrate from a solution in chlorobenzene. Chlorobenzene is chosen since it is known not to have a strong influence on the surface composition [47]. Average thicknesses of the films are measured using a stylus profilometer. A Kratos ES300 electron spectrometer with $\mathrm{Mg} \mathrm{K} \alpha$ X-rays (nonmonochromatic) is used for XPS analysis. A typical sample is a ca. $0.5 \mathrm{~mm}$-thick silicon wafer with dimensions of $4 \mathrm{~mm} \times 12 \mathrm{~mm}$. In the standard geometry the sample accepts $\mathrm{X}$-rays at $45^{\circ}$ and emits photoelectrons at $90^{\circ}$ with respect to its surface plane. The sample can also be rotated to decrease the emission angle (electron take-off angle) in order to enhance surface sensitivity while keeping the X-ray-sample-analyzer angle always at $45^{\circ}$. Calibration of the energy scale was carried out using standard silver and gold samples and referencing to the $C 1$ s peak at $285.0 \mathrm{eV}$. Resolution of the spectrometer was better than $0.9 \mathrm{eV}$ measured in the $\mathrm{Ag} 3 \mathrm{~d}$ peaks and we used standard curve fitting routines with $0.6 \mathrm{eV}$ spin-orbit parameter for the Si $2 \mathrm{p}$ peak.

For probing charging or electrical properties, the sample substrate is subjected either to $\pm 10 \mathrm{~V}$ dc stress or to SQW of $\pm 10 \mathrm{~V}$ amplitude with varying frequencies in the $10^{-3}$ to $10^{3} \mathrm{~Hz}$ range, while recording XPS data.

Samples are electrically connected both from the top (the oxide layer or the polymer film) and also from the bottom (the silicon substrate) to the sample holder, which is electrically isolated from the spectrometer ground, through which the external voltage stress can be applied. For controlling the charging sate of the sample via the applied external voltage stress, a simple hot tungsten filament is placed near the sample. Typically $3.0 \mathrm{~A}$ current is passed from the filament, which is kept ca. $-2 \mathrm{~V}$ with respect to the ground. Typical currents are ca. $10 \mathrm{nA}$ due to photoelectrons going out of the sample into the spectrometer, and ca. $20 \mathrm{nA}$ going into the spectrometer from the X-ray gun and/or stray electrons from the other features like ion-gauge, etc. However, the largest supply of electrons is the filament where the currents as high as $10 \mu \mathrm{A}$ can be obtained when the sample is positively biased $[38,39]$.

\section{Results}

\subsection{Control of charging/discharging properties by application of dc stress}

When an external voltage stress is applied to the sample substrate, while recording an XPS spectrum of a poorly conducting/dielectric material, the binding energies shift in a very non-linear fashion due to variation of the electron current passing through the sample. This is best exemplified by measurements carried out on a very thin $\mathrm{SiO}_{2}$ overlayer $(<10 \mathrm{~nm})$, since the $\mathrm{Si} 2 \mathrm{p}$ peaks of both the oxide and the silicon underlayer can be observed simultaneously as shown in Fig. 1 for a sample with ca. $5 \mathrm{~nm}$ oxide

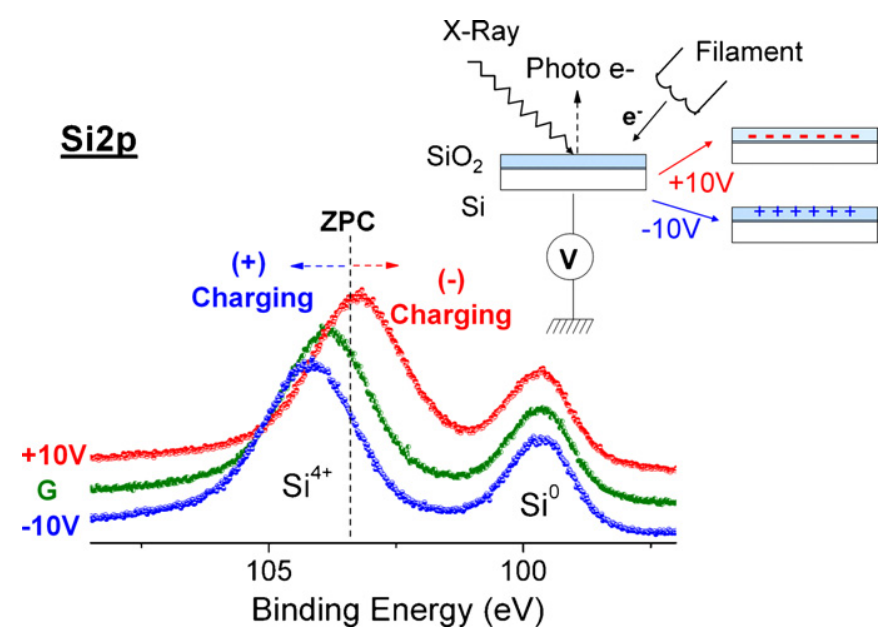

Fig. 1. Si 2p region of the XPS spectrum of a silicon wafer sample containing ca. $5 \mathrm{~nm}$ thermal oxide layer when the sample is grounded and under +10 and $-10 \mathrm{~V}$ external bias, and when it is subjected to additional low-energy electrons from a filament nearby. The inset on the top right depicts the experimental setup.

thickness. As can be seen from the figure the changes in the measured positions of the Si $2 \mathrm{p}$ peaks as a function of the applied potential to the sample of silicon are very different with respect to the polarity of the voltage stress. After correcting for the simple potential shifts, the peak of the underlying substrate $\left(\mathrm{Si}^{0}\right)$ aligns perfectly, however, the peak of the oxide layer $\left(\mathrm{Si}^{4+}\right)$ shifts non-linearly, leading to different measured chemical shifts due to the differences in the electrical potential built-up as a result of charging. The Si $2 p$ binding energies of the substrate and a truly uncharged oxide layer (at the zero-point-charge state, ZPC) are 99.5 and $103.2 \mathrm{eV}$, respectively [24]. Hence, a measured binding energy larger than $103.2 \mathrm{eV}$ corresponds to a positively charged oxide layer, and a smaller value is the sign of a negatively charged oxide layer. Accordingly, as shown in Fig. 1, both in the cases where the sample is grounded and when $\mathrm{a}-10 \mathrm{~V}$ potential is applied externally, the oxide layer is positively charged, due to the uncompensated photoelectrons leaving the sample. Only after application of $+10 \mathrm{~V}$, the oxide layer becomes negatively charged due to a relatively larger electron current flowing through the sample, since more electrons from the filament are attracted to the positively biased sample. As we had shown in our previous time-dependent XPS measurements, the charging state of the oxide layer can easily be controlled by changing the polarity of the external bias, and the fully charged (or discharged) state in the oxide layer is achieved within few seconds [36,38].

\subsection{An artificial dielectric layer: $A u(m)+$ external $R C$}

The simplest model system for relating the experimentally measured charging shifts in the binding energies (BE), and equally important, in the full-widths-at-half maximum (FWHM) of the corresponding peaks, to the charging/discharging capacities, and/or electrical properties of the dielectric sample(s) under consideration, might be considered as the dielectric layer having a series resistance $(R)$ and a parallel capacitance $(C)$. For that reason, we first took a Au metal strip and measured both the BE and the FWHM of the Au 4f spin-orbit doublet, under different experimental conditions as shown in Figs. 2 and 3. First type of measurements involve application of -10 and $+10 \mathrm{~V}$ dc to the sample, which simply shifts the $\mathrm{Au} 4 \mathrm{f}$ position at $84.0 \mathrm{eV}$ to higher and lower $10.0 \mathrm{eV}$ values exactly, since the gold metal is perfectly conducting. However, the shifts are quite asymmetrical when an external series resistance of $1.0 \mathrm{M} \Omega$ value is incorporated into the circuit. Although no significant shift is observed in the case where $-10 \mathrm{~V}$ applied, since 


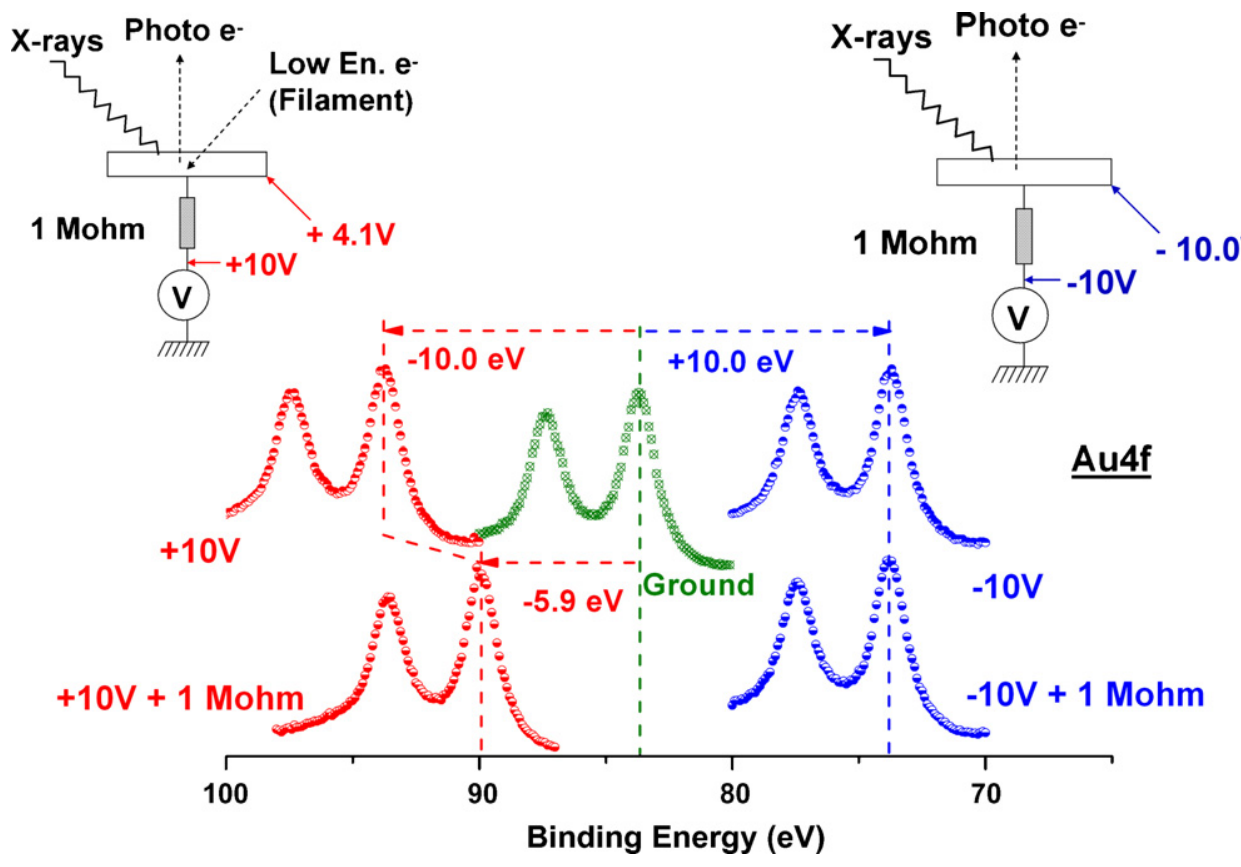

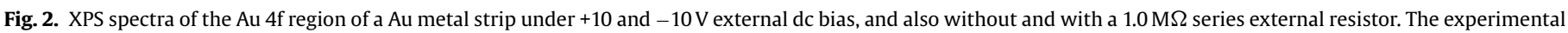
setup is also shown schematically.

all low-energy electrons are effectively repelled, and only emitted ca. $10 \mathrm{nA}$ photoelectrons remain as the current source to yield $10 \mathrm{nA} \times 1 \mathrm{M} \Omega=0.01 \mathrm{eV}$ shift, a significant additional IR potential drop develops as measured from the $4.1 \mathrm{eV}$ difference between the Au $4 \mathrm{f}$ levels with and without the external resistor, when $+10 \mathrm{~V}$ is applied (Fig. 2). This is due to the enhanced low-energy electron current flowing, stemming, mostly from the filament, and passing through the sample. Note that the negative charges causing the shift of $4.1 \mathrm{eV}$ are all accumulated on the external circuit ( $R$ in this case), and as a result the $\mathrm{Au}(\mathrm{m})$ is uniformly shifted, as manifested with no significant changes in the measured FWHM of the Au $4 \mathrm{f}$ peak. This situation is quite different from the case of the $\mathrm{SiO}_{2}$ layer, as will be pointed out below.

The application of an ac stress in the form of square-wave pulses to the $\mathrm{Au}(\mathrm{m})$ sample splits the Au $4 \mathrm{f}$ doublet into two components

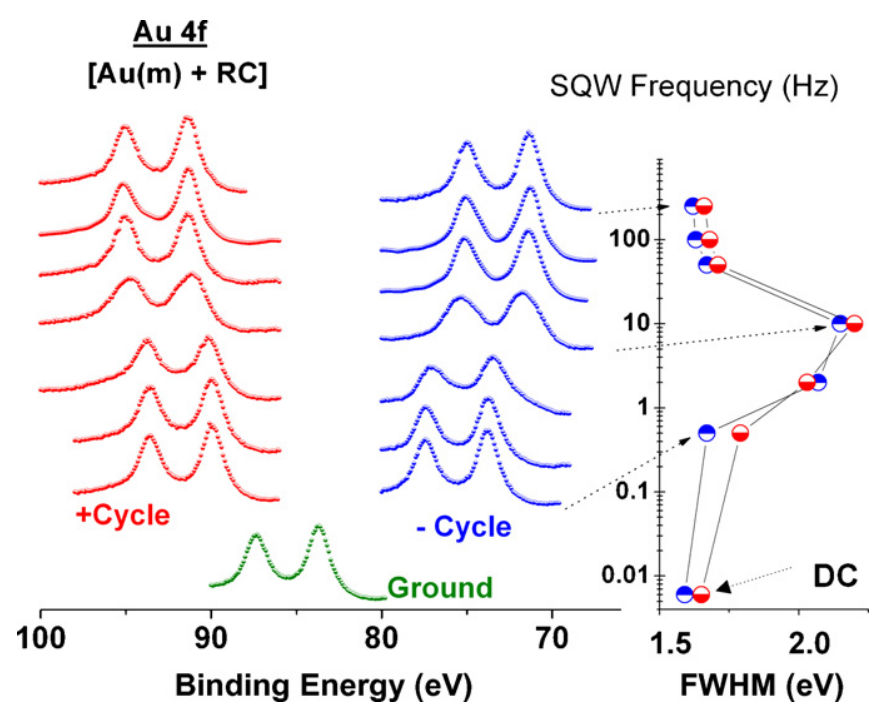

Fig. 3. XPS spectra of the Au $4 \mathrm{f}$ region when the metal is connected externally to a $1.0 \mathrm{M} \Omega$ series resistance and a $56 \mathrm{nF}$ parallel capacitance under various $\mathrm{SQW}$ pulses. Variations in the FWHM of the twinned peaks are also shown on the right hand side. appearing simultaneously at correspondingly -10.0 and $+10.0 \mathrm{eV}$ with respect to its original position, since the sample now spends $50 \%$ of its time at $10.0 \mathrm{~V}$ higher and lower potentials in the negative and the positive cycles, respectively. For the case when $\mathrm{Au}(\mathrm{m})$ is connected externally to a series resistance, $R=1.0 \mathrm{M} \Omega$, and a parallel capacitance, $C=56 \mathrm{nF}$, as shown in Fig. 3, the situation becomes different. The Au $4 \mathrm{f}$ peak is also split but the shifts are not only smaller than $\pm 10.0 \mathrm{eV}$, but they also exhibit strong frequency dependence, due to dynamical charging/discharging through the external RC circuit under the influence of the voltage stimuli. At low frequencies, the difference between the two components is smaller than $20.0 \mathrm{eV}$, and towards higher frequencies the $20.0 \mathrm{eV}$ difference is recovered. Furthermore, the FWHMs of both the positive and negative components go through a maximum around $5 \mathrm{~Hz}$ and starts declining afterwards, resuming their original values at high frequencies. But the measured FWHM values of the positive and the negative components track each other very closely (see also Fig. 7), differing from the case of the $\mathrm{SiO}_{2}$ layer, as will also be pointed out later in the text (see also Fig. 9).

\subsection{An organic dielectric layer: polystyrene (PS)}

When a thin film ( $20 \mathrm{~nm})$ of polystyrene, deposited on a clean silicon wafer by spin coating, is subjected to external dc stress, the position of the $C 1 \mathrm{~s}$ peak also shifts to lower or higher binding energies as displayed in Fig. 4. In this case, although the extent of the charging shift is different, no significant broadening is observed. When the sample is subjected to SQW pulses with varying frequencies, in addition to the charging shifts, variations in the FWHMs are also observed as shown separately in Fig. 8.

\subsection{An inorganic dielectric layer: $10 \mathrm{~nm}$ thermal $\mathrm{SiO}_{2}$ layer}

In Fig. 5, we display the Si $2 \mathrm{p}$ region of the XPS spectra of a Si wafer containing ca. $10 \mathrm{~nm}$ thermal oxide layer, recorded under SQW pulses at three different frequencies, as well as at +10 and $-10 \mathrm{~V}$ dc potentials. In addition to the charging shifts, the observed FWHMs show polarity, as well as frequency dependence, which are displayed separately in Fig. 9. Under $+10 \mathrm{~V}$ dc and also at low fre- 


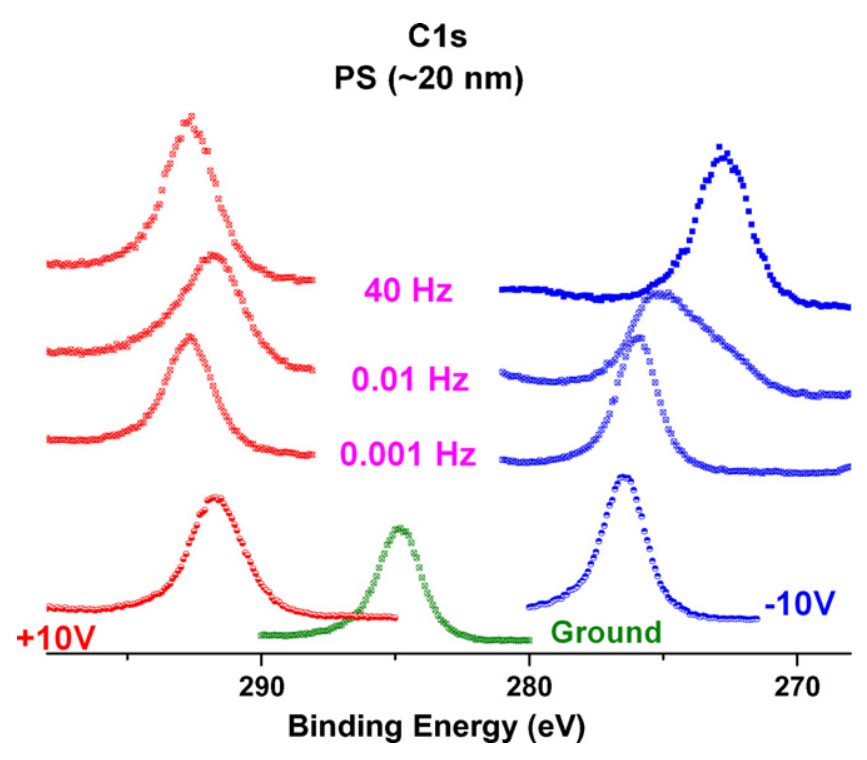

Fig. 4. $C 1$ s region recorded when grounded and under $\pm 10 \mathrm{~V}$ dc external bias, and under SQW pulses of $0.001,0.01$ and $40 \mathrm{~Hz}$ of a ca. $20 \mathrm{~nm}$ PS film on a silicon wafer.

quencies, the negatively charged component is always broader, and becomes narrower only at high frequencies. The positively charged component is narrow at both ends but goes through a maximum around $0.02 \mathrm{~Hz}$. Furthermore, the $\mathrm{O}$ 1s peak exhibits exactly the same frequency dependence both in position and also in FWHM.

\section{The model}

A simple circuit model of a dielectric film having a resistance $R$, and a capacitance $C$ can be constructed as shown in Fig. 6(a), where the electron gun and the photoelectron currents are modeled by a voltage controlled current source (VCCS). The non-linear voltage-current curve for the VCCS is extracted through analysis of a series of XPS measurements by applying DC voltages to the gold sample in series with a known external resistor. The VCCS current-voltage dependence is assumed to be the same for the other samples, and throughout the entire dynamic measurements. The excitation voltage source $V_{E X}(t)$ can be a time varying voltage with any arbitrary shape. In order to calculate the XPS spectra, we

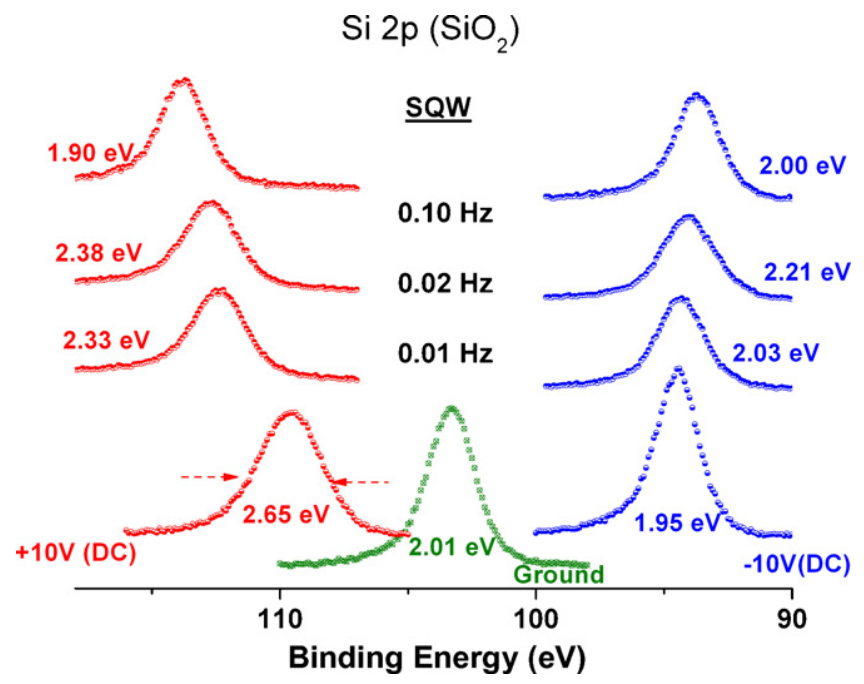

Fig. 5. Si 2 p spectrum of silicon wafer sample containing ca. $10 \mathrm{~nm}$ thermal oxide layer recorded, when grounded and under $\pm 10 \mathrm{~V}$ dc external bias, and when subjected to $10 \mathrm{~V}$ square-wave pulses at $0.01,0.02$ and $0.1 \mathrm{~Hz}$.

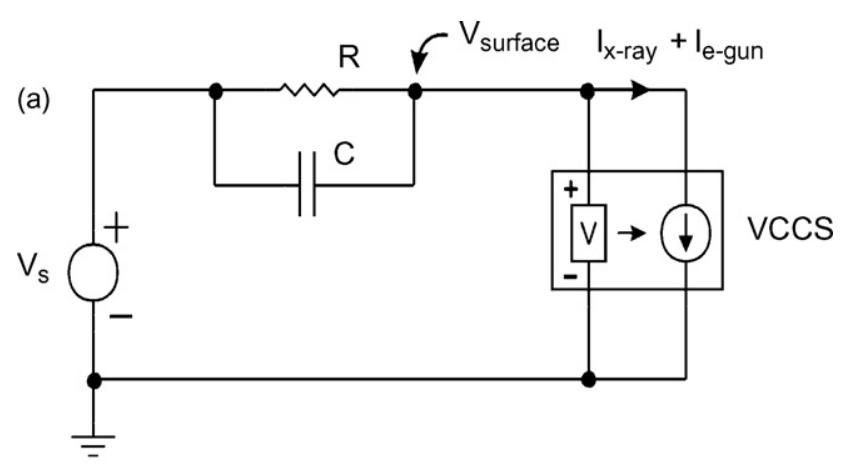

(b)

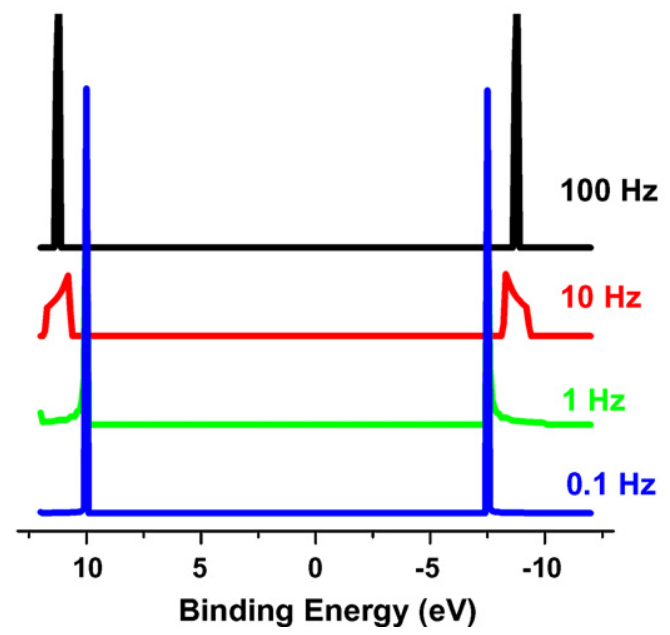

Fig. 6. (a) The circuit used for simulation of the frequency dependence measured and given in Figs. 7-9. (b) Calculated line shapes for the $1.0 \mathrm{M} \Omega$ and $56 \mathrm{nF}$ RC circuit.

need to solve the differential equation describing the change of the surface potential $V_{S}(t)$ given by

$C \frac{d\left(V_{S}-V_{E X}\right)}{d t}+\frac{V_{S}-V_{E X}}{R}+I_{S}\left(V_{S}\right)=0$

where $R$ and $C$ are, respectively, the effective resistance and the capacitance between the surface and the source as shown in Fig. 6(a), and $I_{S}\left(V_{S}\right)$ is the surface potential dependent current of the electron flood-gun. Solutions can be obtained by numerical integration for arbitrary excitations $V_{E X}(t)$. For a periodic excitation of period $T$, one can calculate a line-shape function that gives the intensity of the XPS signal at a voltage shift of $v$ as

$g(v)=\frac{1}{T} \int_{0}^{T} \delta\left[V_{S}(t)-v\right] d t$

Note that $\int_{-\infty}^{\infty} g(v) d v=1$ and hence the line-shape function is properly normalized. The XPS spectrum recorded by the spectrometer $S_{X P S}(v)$ can now be calculated by convolution of the line-shape function $g(v)$ with the original spectrum $S_{0}(v)$ obtained by grounding the sample:

$S_{X P S}(v)=\int_{-\infty}^{\infty} g(v-\lambda) S_{0}(\lambda) d \lambda$.

By solving numerically Eq. (1) for the values of $R=1.0 \mathrm{M} \Omega$ and $C=56 \mathrm{nF}$ for the square-wave excitation, we calculate the lineshape functions through Eq. (2) as shown in Fig. 6(b). It can be seen that, depending only on the period of the excitation and charge-discharge time constants of the sample system, the left and right peaks of the line-shape function shift differently. If the excitation signal period is much higher than the sample time constants, we are working in a regime similar to $\mathrm{dc}$ In this case, the peaks are 


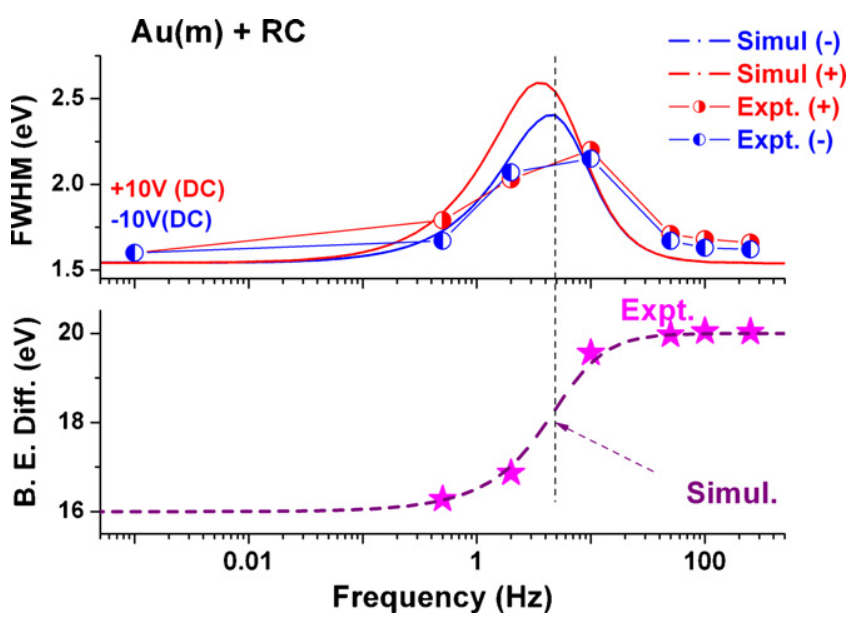

Fig. 7. Frequency dependences of the measured FWHM and binding energy difference of Au metal with a $1.0 \mathrm{M} \Omega$ series resistance and a $56 \mathrm{nF}$ parallel capacitance using the model described in Fig. 6. The solid lines are theoretically calculated and the line + symbol are the experimentally measured values plotted in a semilog fashion.

shifted almost to the positions that would be obtained by applying -10 or $+10 \mathrm{~V}(\mathrm{dc})$ to the sample, respectively. Also, the broadening is almost zero. If the excitation frequency is high, the average surface potential is shifted due to the non-linear nature of the VCCS, but the separation between the left and right peaks is closer to $20 \mathrm{~V}$.

\section{Discussion and conclusions}

When applied to nonconducting dielectric samples, our method measures an effective time constant comprised of an effective resistance and capacitance of the films under X-rays and low-energy electrons exposure. The charging and discharging of the films are non-linearly dependent on the surface electrical field created since they are described by tunneling or hopping processes. The frequency dependence of the difference in the binding energy between the left $(+$ cycle) and the right (-cycle) peaks varies like an S-type curve. Also, there are variations in the broadening of the peaks. It is seen through calculations that, for the square-wave case, the peaks have maximum broadening as $1 / 4$ of the period of excitation coincides with the charging/discharging time constant of the sample, which corresponds to the inflection point of the S-curve. As we have discussed and advocated in our previous work, we believe that this S-curve is material specific.

For the case of the artificial dielectric of $\mathrm{Au}(\mathrm{m})+\mathrm{RC}$, the time constant should be $0.056 \mathrm{~s}(1.0 \mathrm{M} \Omega \times 56 \mathrm{nF})$, and this would correspond to $4.5 \mathrm{~Hz}[1 / 4 \times 1 /(0.056 \mathrm{~s})]$ for the maximum broadening, which matches the experimental results perfectly, as depicted in Fig. 7. Similar measurements for the $20 \mathrm{~nm}$ PS film yield the time constant of $17 \mathrm{~s}$ using the fit to the experimental frequency data in the binding energy difference, and determine the inflection point at $0.015 \mathrm{~Hz}$, as shown in Fig. 8. As was shown in our previous work, a thicker PS (ca. $55 \mathrm{~nm}$ ) film exhibits larger binding energy shifts, but the frequency dependence is surprisingly similar to that of the $20 \mathrm{~nm}$ PS film, as judged by the coincidence of their inflection points [40]. In the same work, we also showed that another organic dielectric PMMA film exhibited a different frequency dependence, which encouraged us to advocate that this measured frequency dependence is material specific.

In the case of the $10 \mathrm{~nm} \mathrm{SiO}$ the time constant is estimated as $12 \mathrm{~s}$, again using the experimentally determined inflection point in the binding energy shifts as shown in Fig. 9. Accordingly the maximum broadening is predicted to be around $0.02 \mathrm{~Hz}$, again in perfect agreement with experimental data measured for the -cycle. However, as also mentioned above the extra broadenings appearing

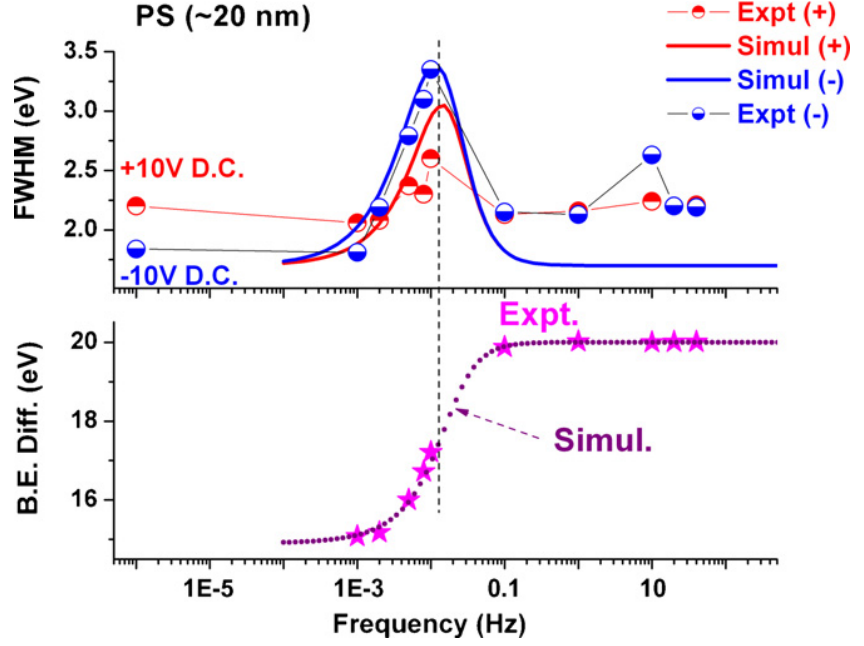

Fig. 8. Measured and calculated frequency dependences of the measured FWHM and binding energy difference of the $20 \mathrm{~nm}$ PS sample.

when the dielectric bears negative charging (at low frequencies and only on the +cycle) cannot be accounted for by our simple model.

The simple RC model can be replaced with a more accurate model that takes into account of the distribution of charge-traps. However, the ultimate model has to include E-field and position dependent charge-discharge rates as well as local density of trap states. For the case of the $\mathrm{SiO}_{2}$ layer, the charging and discharging processes we probe are related with trapping and detrapping of the holes created in the valence band of the oxide following the very fast $\left(<10^{-12} \mathrm{~s}\right)$ photoemission process [24]. Therefore, the time constants we measure are comparable to the time constants derived by time-dependent leakage currents determined for MOS systems under X-ray exposure [6-8] and/or using scanning capacitance microscopy [9]. For the case of organic dielectric materials, the situation is even less understood, and thus more important $[4,5]$.

In conclusion, we show that by application of time-dependent voltage stimuli, in the form of square waves, to dielectric materials, when recording their XPS spectra, it is possible to probe the dynamics of their charging/discharging properties. The method twins each peak, and the measured binding energy difference between the twinned peaks, and the variations in the FWHM exhibit strong

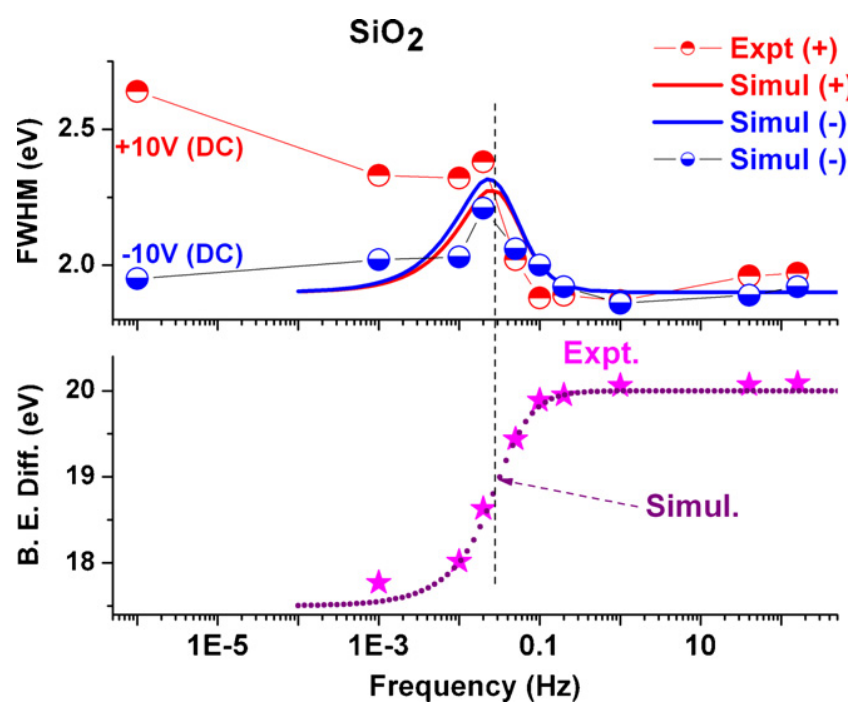

Fig. 9. Measured and calculated frequency dependences of the measured FWHM and binding energy difference of the silicon sample with a ca. $10 \mathrm{~nm}$ thermal oxide layer. 
frequency dependence. We believe that through careful analyses of these frequency dependencies it is possible to extract various material-specific dielectric properties, like effective resistance and capacitance, dielectric breakdown behavior, etc.

\section{Acknowledgements}

This work was partially supported by TUBA (Turkish Academy of Sciences) and TUBITAK (The Scientific and Technological Research Council of Turkey) through the Grants No.: $106 \mathrm{~T} 409$.

\section{References}

[1] S.M. Sze, Physics of Semiconductor Devices, Wiley, New York, 1981.

[2] D.A. Muller, T. Sorsch, S. Moccio, F.H. Baumann, K. Evans-Lutterodt, G. Timp, Nature 399 (1999) 758-761.

[3] G.M. Sessler, R. Gerhard-Multhaupt (Eds.), Electrets, 3rd ed., Morgan Hill, 1998.

[4] L.S. McCarty, G.M. Whitesides, Angew. Chem. Int. Ed. 47 (2008) 2188-2207.

[5] C. Liu, A.J. Bard, Nat. Mater. 7 (2008) 505-509.

[6] O.L. Curtis Jr., J.R. Srour, J. Appl. Phys. 48 (1977) 3819-3828.

[7] R.C. Hughes, Phys. Rev. B 15 (1977) 2012-2020.

[8] T. Wang, T.E. Cheng, L.P. Chiang, C.H. Wang, N.K. Zons, C. Huang, IEEE Trans, Electron. Devices 45 (1998) 1511-1517.

[9] C.J. Kang, G.H. Buh, S. Lee, C.K. Kim, K.M. Mang, C. Im, Y. Kuk, Appl. Phys. Lett. 74 (1999) 1815-1817.

[10] H. Hovel, B. Grimm, M. Pollmann, B. Reihl, Phys. Rev. Lett. 81 (1998) 4608-4611.

[11] M. Marsi, R. Belkhou, C. Grupp, G. Panaccionne, A. Taleb-Ibrahimi, L. Nahon, D. Garzella, D. Nutarelli, E. Renault, R. Roux, M.E. Couprie, M. Billardon, Phys. Rev. B 61 (2000) R5070-R5073.

[12] M. Bauer, C. Lei, K. Read, R. Tobey, J. Gland, M.M. Murnane, H.C. Kapteyn, Phys. Rev. Lett. 87 (2001) (article no.: 025501).

[13] J.P. Countour, G. Mouvier, Chem. Phys. Lett. 33 (1975) 237-240.

[14] T.L. Barr, Crit. Rev. Anal. Chem. 22 (1991) 229-325.

[15] W.M. Lau, J. Appl. Phys. 65 (1989) 2047-2052.

[16] A. Fernandez, J.P. Espinos, D. Leinen, A.R. Gonzales-Elipe, J.M. Sanz, Surf. Interface Anal. 22 (1994) 111-114.

[17] B.J. Tielsch, J.E. Fulghum, Surf. Interface Anal. 25 (1997) 904-912.
[18] N.J. Havercroft, P.M.A. Sherwood, Surf. Interface Anal. 29 (2000) 232-240.

[19] D. Briggs, M.P. Seah, Practical Surface Analysis. Part I. Auger and X-ray Photoelectron Spectroscopy, 2nd ed., John Wiley \& Sons Ltd., England, 1996.

[20] J. Cazaux, J. Electron Spectrosc. Relat. Phenom. 105 (1999) 155-185.

[21] J. Cazaux, J. Electron Spectrosc. Relat. Phenom. 113 (2000) 15-33.

[22] M. Grimm, B. Langer, S. Schlemmer, T. Lischke, U. Becker, W. Widdra, D. Gerlich, R. Flesch, E. Ruhl, Phys. Rev. Lett. 96 (2006) (article no.: 066801)

[23] W.M. Lau, X.W. Wu, Surf. Sci. 245 (1991) 345-352.

[24] S. Iwata, A. Ishizaka, J. Appl. Phys. 79 (1996) 6653-6713.

[25] H. Doron-Mor, A. Hatzor, A. Vaskevich, T. van der Boom-Moav, A. Shanzer, I. Rubinstein, H. Cohen, Nature 406 (2000) 382-385.

[26] D.R. Daer, M.H. Engelhard, D.J. Gaspar, A.S. Lea, C.F. Windisch Jr., Surf. Interface Anal. 33 (2002) 781-790.

[27] K. Shabtai, I. Rubinstein, S.R. Cohen, H. Cohen, J. Am. Chem. Soc. 122 (2000) 4959-4962.

[28] H. Cohen, Appl. Phys. Lett. 85 (2004) 1271-1273.

[29] M. Dubey, I. Gouzman, S.L. Bernasek, J. Schwartz, Langmuir 22 (2006) 4649-4653.

[30] I. Gouzman, M. Dubey, M.D. Carolus, J. Schwartz, S.L. Bernasek, Surf. Sci. 600 (2006) 773-781.

[31] A.Vilan, T.A. Bendikov, H.J. Cohen, Electron Spectrosc. Relat. Phenom. 162 (2008) 99-105.

[32] A. Islam, M. Mukherjee, J. Phys. Chem. B 112 (2008) 8523-8529.

[33] S. Suzer, Anal. Chem. 75 (2003) 7026-7029.

[34] F. Karadas, G. Ertas, S. Suzer, J. Phys. Chem. B 108 (2004) 1515-1518.

[35] G. Ertas, S. Suzer, Surf. Interface Anal. 36 (2004) 619-623.

[36] U.K. Demirok, G. Ertas, S. Suzer, J. Phys. Chem. B 108 (2004) 5179-5181.

[37] G. Ertas, U.K. Demirok, S. Suzer, Appl. Surf. Sci. 248 (2005) 12-15.

[38] G. Ertas, U.K. Demirok, A. Atalar, S. Suzer, Appl. Phys. Lett. 86 (2005) (article no.: 183110).

[39] E. Karabudak, U.K. Demirok, S. Suzer, Surf. Sci. Lett. 600 (2006) L12-L14.

[40] H. Sezen, G. Ertas, A. Dana, S. Suzer, Macromolecules 40 (2007) 4109-4112.

[41] S. Suzer, A. Dana, J. Phys. Chem. B 110 (2006) 19112-19115.

[42] T.O. Tasci, E. Atalar, U.K. Demirok, S. Suzer, Surf. Sci. 602 (2008) 365-368.

[43] Y. Feldman, A. Zak, R. Tenne, H. Cohn, J. Vac. Sci. Technol. A 21 (2003) 17521757.

[44] Y. Hagimoto, H. Fujioka, M. Oshima, Appl. Phys. Lett. 77 (2000) 4175-4177.

[45] K. Hirose, K. Sakano, K. Takahashi, T. Hattori, Surf. Sci. 507-510 (2002) 906-910.

[46] A.J. Bard, L.R. Faulkner, Electrochemical Methods, Wiley, New York, 2001.

[47] S. Walheim, M. Boltau, J. Mlynek, G. Krausch, U. Steiner, Macromolecules 30 (1997) 4995-5003. 